\title{
Linear beam optics in solenoidal channels
}

\author{
G. Franchetti \\ CERN, CH-1211, Geneva 23, Switzerland and GSI Darmstadt, D-64291 Darmstadt, Germany
}

(Received 7 March 2001; published 20 July 2001)

\begin{abstract}
In this paper we study beam transport through a straight solenoidal channel using the single-particle and linear optics approach. We derive the single-particle invariants and show their use in an extended CourantSnyder theory for a solenoidal coupled system. Matching between solenoidal channels and between solenoidal and quadrupolar channels is discussed. We give envelope solutions and illustrate them with some numerical examples.
\end{abstract}

DOI: 10.1103/PhysRevSTAB.4.074001

PACS numbers: 41.85.Ja

\section{INTRODUCTION}

For muon colliders [1,2] and neutrino sources [3], lossless beam transport through solenoidal channels is an important issue which requires a careful design. Technical hardware limits and cost control impose further constraints which make the design challenging. Typically, the muon beam exhibits a very large emittance which puts part of the beam in a nonparaxial beam optics regime. The problem requires a full $6 \mathrm{D}$ description: however, as a first step, one can consider the beam in a linear paraxial regime and use an analytical solution of the single particle dynamics. This method gives important guidelines for the initial design; further optimization with numerical integration $[4,5]$ is required for final performance evaluation. Here we present the analytical solution of the single particle motion in a straight solenoidal transport channel using the following approximations: (i) the motion is paraxial and (ii) the equations of motion are linear.

\section{SINGLE-PARTICLE SOLUTION AND INVARIANTS}

Let us consider the equations of motion for a particle in a solenoidal channel in the linear approximation [6]

$$
\begin{aligned}
& x^{\prime \prime}-S y^{\prime}-\frac{1}{2} S^{\prime} y=0, \\
& y^{\prime \prime}+S x^{\prime}+\frac{1}{2} S^{\prime} x=0,
\end{aligned}
$$

where $S=q[\mathrm{C}] B_{s}(s)[\mathrm{T}] / p[\mathrm{~kg} \mathrm{~m} / \mathrm{s}]$. Here, $q$ is charge of the particle, $B_{s}$ is the longitudinal magnetic field, and $p$ is the longitudinal momentum. With ' we denote the space derivative $d / d s$. By using Eqs. (1), we assume that $B_{s}$ has no radial dependence on the transverse (radial) position of the particle. General methods [7,8] for linear coupled particle dynamics have been proposed. Here we discuss a general method to solve Eqs. (1). Following $[6,8]$ we define the complex variable $z=x+i y$ so that the pair of differential equations [Eqs. (1)] becomes the single complex differential equation

$$
z^{\prime \prime}+i S z^{\prime}+\frac{i}{2} S^{\prime} z=0
$$

The complex dynamic variables can be written as $\mathbf{z}=$ $\left(z, z^{\prime}\right)$. It is well known that in the Larmor frame the equations of motion decouple into two Hill's differential equations. Following $[6,8]$ the Larmor rotation is given by the transformation $w=z e^{i \phi}$, where $\phi$ is the Larmor angle $\phi(s)=\int_{0}^{s} S(t) / 2 d t$. Defining the Larmor complex dynamic variables $\mathbf{w}=\left(w, w^{\prime}\right)$, the transformation from $\mathbf{w}$ to $\mathbf{z}$ is given by

$$
\mathbf{z}=\Lambda \mathbf{w}, \quad \text { with } \Lambda=e^{-i \phi}\left(\begin{array}{cc}
1 & 0 \\
-i \phi^{\prime} & 1
\end{array}\right) .
$$

Since $S$ is real and $w=w_{r}+i w_{i}$, the equations of motion on the Larmor frame can be decomposed into two decoupled Hill's differential equations,

$$
w_{r}^{\prime \prime}+\phi^{\prime 2} w_{r}=0, \quad w_{i}^{\prime \prime}+\phi^{\prime 2} w_{i}=0 .
$$

The coefficient $\phi^{\prime 2}$ is the square of the solenoid strength which always gives a focusing character to Eqs. (4). Each of these equations can be solved with the method used in the Courant-Snyder theory [9]. The dynamic variables of Eqs. (4), $\left(w_{r}, w_{r}^{\prime}\right)$ and $\left(w_{i}, w_{i}^{\prime}\right)$, are transported by the same map which has the standard form [10]

$$
\mathrm{M}=\mathrm{T}_{1} \mathrm{RT}_{0}^{-1},
$$

where

$$
\mathrm{R}=\left(\begin{array}{cc}
\mathrm{c} & \mathrm{s} \\
-\mathrm{s} & \mathrm{c}
\end{array}\right), \quad \mathrm{T}_{j}=\left(\begin{array}{cc}
\sqrt{\beta_{j}} & 0 \\
-\frac{\alpha_{j}}{\sqrt{\beta_{j}}} & \frac{1}{\sqrt{\beta_{j}}}
\end{array}\right),
$$

with $\mathrm{C}=\cos \psi, \mathrm{S}=\sin \psi$. Here $j=0,1$, where the index 0 means particle at $s=0$, and the index 1 means particle at $s$. The Twiss parameters $\beta, \alpha$, and $\psi$ are given by

$$
\begin{aligned}
& \frac{1}{2} \beta \beta^{\prime \prime}-\frac{1}{4} \beta^{\prime 2}+\frac{S^{2}}{4} \beta^{2}=1, \\
& \alpha=-\frac{\beta^{\prime}}{2}, \quad \psi=\int_{0}^{s} \frac{1}{\beta(t)} d t,
\end{aligned}
$$


with $\alpha_{0}, \beta_{0}$ the arbitrary initial Twiss parameters. Recombining the two vectorial representations in a complex vectorial form, the transfer map in $\left\{w, w^{\prime}\right\}$ becomes $\mathbf{w}_{1}=M \mathbf{w}_{0}$. The explicit form of the transport matrix is

$$
\mathrm{M}=\left(\begin{array}{cc}
\sqrt{\frac{\beta_{1}}{\beta_{0}}}\left(\mathrm{c}+\alpha_{0} \mathrm{~s}\right) & \sqrt{\beta_{1} \beta_{0}} \mathrm{~s} \\
\frac{\left(\alpha_{0}-\alpha_{1}\right) \mathrm{c}-\left(1+\alpha_{1} \alpha_{0}\right) \mathrm{s}}{\sqrt{\beta_{1} \beta_{0}}} & \sqrt{\frac{\beta_{0}}{\beta_{1}}}\left(\mathrm{c}-\alpha_{1} \mathrm{~s}\right)
\end{array}\right) .
$$

Now we can use the transformation Eq. (3) to rewrite the map $\mathrm{M}$ in the frame $\left\{z, z^{\prime}\right\}$. Multiplying $\mathbf{w}_{1}=\mathbf{M} \mathbf{w}_{0}$ by $\Lambda_{1}$ and substituting $\mathbf{w}_{0}=\Lambda_{0}^{-1} \mathbf{z}_{0}$, we find that in $\left\{z, z^{\prime}\right\}, \mathrm{M}$ becomes $L=\Lambda_{1} M \Lambda_{0}^{-1}=\hat{\mathrm{T}}_{1} \mathrm{R} \hat{T}_{0}^{-1}$ where $\hat{\mathrm{T}}=\Lambda \mathrm{T}$. Explicitly,

$$
\hat{\mathrm{T}}=e^{-i \phi}\left(\begin{array}{cc}
\sqrt{\hat{\beta}} & 0 \\
-\frac{\hat{\alpha}}{\sqrt{\hat{\beta}}} & \frac{1}{\sqrt{\hat{\beta}}}
\end{array}\right) .
$$

The hat $\left({ }^{\wedge}\right)$ Twiss parameters are defined as $\hat{\beta}=\beta$, $\hat{\alpha}=$ $\alpha+i \beta \phi^{\prime}$, and $\hat{\gamma}=\left(1+\hat{\alpha}^{2}\right) / \hat{\beta}$. By using the formal identity of Eq. (9) with Eq. (6) right, it is straightforward to find

$$
\mathrm{L}=e^{-i \phi_{1}}\left(\begin{array}{cc}
\sqrt{\frac{\hat{\beta}_{1}}{\hat{\beta}_{0}}}\left(\mathrm{c}+\hat{\alpha}_{0} \mathrm{~s}\right) & \sqrt{\hat{\beta}_{1} \hat{\beta}_{0}} \mathrm{~s} \\
\frac{\left(\hat{\alpha}_{0}-\hat{\alpha}_{1}\right) \mathrm{c}-\left(1+\hat{\alpha}_{1} \hat{\alpha}_{0}\right) \mathrm{s}}{\sqrt{\hat{\beta}_{1} \hat{\beta}_{0}}} & \sqrt{\frac{\hat{\beta}_{0}}{\hat{\beta}_{1}}}\left(\mathrm{c}-\hat{\alpha}_{1} \mathrm{~s}\right)
\end{array}\right) .
$$

The solenoidal channel transport map in $\left\{z, z^{\prime}\right\}$ finally reads $\mathbf{z}_{1}=L \mathbf{z}_{0}$. In order to transform the map $L$ defined in $\left\{z, z^{\prime}\right\}$ to the map $\Sigma(\mathrm{L})$ defined in the laboratory frame $\left\{x, x^{\prime}, y, y^{\prime}\right\}$, we need to use the definition $z=x+i y$ which leads to

$$
\Sigma(\mathrm{L})=\left(\begin{array}{cc}
\operatorname{ReL} & -\operatorname{ImL} \\
\operatorname{ImL} & \operatorname{ReL}
\end{array}\right) .
$$

From Eq. (10) it is straightforward to find that ReL $=$ $\mathfrak{c}_{1} \mathrm{M}+\mathfrak{s}_{1}\left(\mathrm{M}_{\mathrm{i} 1}+\mathrm{M}_{\mathrm{i} 2}\right)+\mathfrak{c}_{1} \mathrm{M}_{\mathrm{r}}$, and $-\mathrm{ImL}=\mathfrak{s}_{1} \mathrm{M}-$ $\mathfrak{c}_{1}\left(\mathrm{M}_{\mathrm{i} 1}+\mathrm{M}_{\mathrm{i} 2}\right)+\mathfrak{s}_{1} \mathrm{M}_{\mathrm{r}}$, with $\mathfrak{s}_{1}=\sin \phi_{1}, \mathfrak{c}_{1}=\cos \phi_{1}$, $M$ given by Eq. (8), and

$$
\begin{aligned}
\mathrm{M}_{\mathrm{i} 1} & =\left(\begin{array}{cc}
0 \\
\sqrt{\frac{\beta_{0}}{\beta_{1}}} \phi_{0}^{\prime}\left(\mathrm{c}-\alpha_{1} \mathrm{~s}\right)-\sqrt{\frac{\beta_{1}}{\beta_{0}}} \phi_{1}^{\prime}\left(\mathrm{c}+\alpha_{0} \mathrm{~s}\right) & 0
\end{array}\right), \\
\mathrm{M}_{\mathrm{i} 2} & =\left(\begin{array}{cc}
\sqrt{\beta_{1} \beta_{0}} \phi_{0}^{\prime} \mathrm{s} & 0 \\
0 & -\sqrt{\beta_{1} \beta_{0}} \phi_{1}^{\prime} \mathrm{s}
\end{array}\right), \\
\mathrm{M}_{\mathrm{r}} & =\left(\begin{array}{cc}
0 & 0 \\
\phi_{0}^{\prime} \phi_{1}^{\prime} \sqrt{\beta_{1} \beta_{0}} \mathrm{~s} & 0
\end{array}\right) .
\end{aligned}
$$

Equation (11) is the transfer map which transports the initial particle coordinate $\left(x_{0}, x_{0}^{\prime}, y_{0}, y_{0}^{\prime}\right)$ at $s=0$ to $\left(x, x^{\prime}, y, y^{\prime}\right)$ at the longitudinal position $s$.

In order to recover the usual invariants we consider the motion in the frame $\left\{u_{1}, u_{2}\right\}$ defined by the transformation
$\mathbf{u}=\hat{\mathrm{T}}^{-1} \mathbf{z}$, with $\mathbf{u}=\left(u_{1}, u_{2}\right)$. In the frame $\left\{u_{1}, u_{2}\right\}$ the motion becomes a pure rotation, i.e., $\mathbf{u}_{1}=R \mathbf{u}_{0}$. Decomposing it into the two complex components we find

$$
\mathbf{u}_{1 r}=\mathrm{R} \mathbf{u}_{0 r}, \quad \mathbf{u}_{1 i}=\mathrm{R} \mathbf{u}_{0 i},
$$

where $\mathbf{u}_{j r}=\left(u_{j r 1}, u_{j r 2}\right), \mathbf{u}_{j i}=\left(u_{j i 1}, u_{j i 2}\right)$, with $j=0,1$. Equations (13) show the existence of the two invariants $\epsilon_{1}=u_{1 r 1}^{2}+u_{1 r 2}^{2}$ and $\epsilon_{2}=u_{1 i 1}^{2}+u_{1 i 2}^{2}$. With the proper parametrization of $\mathbf{u}$ the two components of $\mathbf{u}_{1}$ become

$$
\begin{aligned}
& u_{1}=\sqrt{\epsilon_{1}} \sin \left(\psi+\delta_{1}\right)+i \sqrt{\epsilon_{2}} \sin \left(\psi+\delta_{2}\right), \\
& u_{2}=\sqrt{\epsilon_{1}} \cos \left(\psi+\delta_{1}\right)+i \sqrt{\epsilon_{2}} \cos \left(\psi+\delta_{2}\right),
\end{aligned}
$$

where $\delta_{1}, \delta_{2}$, the initial phases of the particle, are the other two invariants. By using $\mathbf{u}=\hat{\mathrm{T}}^{-1} \mathbf{z}$ we can write the invariants in the $\left\{z, z^{\prime}\right\}$ frame and finally in the lab frame $\left\{x, x^{\prime}, y, y^{\prime}\right\}$. Since the invariant $\epsilon_{1}, \epsilon_{2}$ exhibits a dependence from $\cos \psi$ and $\sin \psi$, we can try to use combinations of $\epsilon_{1}, \epsilon_{2}$ in order to obtain another invariant of simpler form. An appropriate choice is $\epsilon_{1}+\epsilon_{2}$, and we obtain

$$
\epsilon_{1}+\epsilon_{2}=\epsilon_{x}+\epsilon_{y}+\beta \phi^{\prime 2}\left(x^{2}+y^{2}\right)+2 \beta \phi^{\prime} L_{z},
$$

where $\quad \boldsymbol{\epsilon}_{x}=\gamma x^{2}+2 \alpha x x^{\prime}+\beta x^{\prime 2}, \quad \boldsymbol{\epsilon}_{y}=\gamma y^{2}+$ $2 \alpha y y^{\prime}+\beta y^{\prime 2}$ are the usual horizontal and vertical Courant-Snyder invariants, and $L_{z}=x y^{\prime}-y x^{\prime}$ is proportional to the mechanical angular momentum. From Eqs. (14) we find another invariant which is a combination of $\epsilon_{1}, \epsilon_{2}$ : in fact, calculating $\operatorname{Re} u_{1} \operatorname{Im} u_{2}-\operatorname{Im} u_{1} \operatorname{Re} u_{2}$ we obtain

$$
\sqrt{\epsilon_{1} \epsilon_{2}} \sin \left(\delta_{1}-\delta_{2}\right)=\phi^{\prime}\left(x^{2}+y^{2}\right)+L_{z} .
$$

From Eqs. (15) and (16), given the particle coordinates $x, x^{\prime}, y, y^{\prime}$ and $\delta_{1}, \delta_{2}$, we can compute $\epsilon_{1}, \epsilon_{2}$ less an inversion $\epsilon_{1} \leftrightarrow \epsilon_{2}$. The physical interpretation of Eq. (16) is the following: since in Eqs. (1) the magnetic field is assumed independent of the particle's transverse position, $\phi^{\prime} r^{2}=(q \Psi / 2 \pi) / p$, where $\Psi$ is the magnetic flux through the circular orbit of radius $r$. The sum of $L_{z}$ and $\phi^{\prime} r^{2}$ is proportional to the canonical angular momentum $p_{\theta}$, i.e., $\phi^{\prime} r^{2}+L_{z}=p_{\theta} / p$. Since $p$ is constant, Eq. (16) is just a reformulation of the well-known Busch's theorem, i.e., of the conservation of $p_{\theta}$ for the dynamical systems with cylindrical symmetry [11].

Since the components of $\mathbf{u}_{1}$ give the particle coordinates in a generic longitudinal position during the motion, Eqs. (14) can be used to find the particle coordinates in the frame $\left\{z, z^{\prime}\right\}$. By using $\mathbf{z}_{1}=\hat{\mathrm{T}}_{1} \mathbf{u}_{1}$, remembering the definition $z=x+i y$ and dropping the index 1 (= final position), the solution of Eqs. (1) is 


$$
\begin{gathered}
x=\sqrt{\beta \epsilon_{1}} \mathrm{~s}_{1} \mathfrak{c}+\sqrt{\beta \epsilon_{2}} \mathrm{~s}_{2} \mathfrak{S}, \quad y=\sqrt{\beta \epsilon_{2}} \mathrm{~s}_{2} \mathfrak{c}-\sqrt{\beta \epsilon_{1}} \mathrm{~s}_{1} \mathfrak{S}, \\
x^{\prime}=\sqrt{\frac{\epsilon_{1}}{\beta}}\left(\mathrm{c}_{1} \mathfrak{c}-\alpha \mathrm{s}_{1} \mathfrak{c}-\beta \phi^{\prime} \mathrm{s}_{1} \mathfrak{S}\right)+\sqrt{\frac{\epsilon_{2}}{\beta}}\left(\mathrm{c}_{2} \mathfrak{S}-\alpha \mathrm{s}_{2} \mathfrak{S}+\beta \phi^{\prime} \mathrm{s}_{2} \mathfrak{c}\right), \\
y^{\prime}=\sqrt{\frac{\epsilon_{1}}{\beta}}\left(-\mathrm{c}_{1} \mathfrak{g}+\alpha \mathrm{s}_{1} \mathfrak{g}-\beta \phi^{\prime} \mathrm{s}_{1} \mathfrak{c}\right)+\sqrt{\frac{\epsilon_{2}}{\beta}}\left(\mathrm{c}_{2} \mathfrak{c}-\alpha \mathrm{s}_{2} \mathfrak{c}-\beta \phi^{\prime} \mathrm{s}_{2} \mathfrak{g}\right),
\end{gathered}
$$

where $\mathrm{s}_{1}=\sin \left(\psi+\delta_{1}\right), \quad \mathrm{C}_{1}=\cos \left(\psi+\delta_{1}\right), \quad \mathrm{s}_{2}=$ $\sin \left(\psi+\delta_{2}\right), \quad \mathrm{c}_{2}=\cos \left(\psi+\delta_{2}\right), \quad \mathfrak{g}=\sin \phi, \quad$ and $\mathfrak{c}=$ $\cos \phi$. Since $\phi(0)=0$, the relations between the initial particle coordinates $x_{0}, x_{0}^{\prime}, y_{0}, y_{0}^{\prime}$ and the invariants $\epsilon_{1}, \epsilon_{2}, \delta_{1}, \delta_{2}$ are

$$
\begin{aligned}
& x_{0}=\sqrt{\beta \epsilon_{1}} \sin \delta_{1}, \\
& y_{0}=\sqrt{\beta \epsilon_{2}} \sin \delta_{2}, \\
& x_{0}^{\prime}=\sqrt{\frac{\epsilon_{1}}{\beta}}\left(\cos \delta_{1}-\alpha \sin \delta_{1}\right)+\sqrt{\beta \epsilon_{2}} \phi^{\prime} \sin \delta_{2}, \\
& y_{0}^{\prime}=-\sqrt{\beta \epsilon_{1}} \phi^{\prime} \sin \delta_{1}+\sqrt{\frac{\epsilon_{2}}{\beta}}\left(\cos \delta_{2}-\alpha \sin \delta_{2}\right) .
\end{aligned}
$$

From these equations we can derive the value of the two invariants $\epsilon_{1}, \epsilon_{2}$. We find

$$
\begin{aligned}
& \epsilon_{1}=\epsilon_{x 0}+\phi^{\prime 2} \beta y_{0}^{2}-2 \alpha \phi^{\prime} x_{0} y_{0}-2 \beta \phi^{\prime} y_{0} x_{0}^{\prime}, \\
& \epsilon_{2}=\epsilon_{y 0}+\phi^{\prime 2} \beta x_{0}^{2}+2 \alpha \phi^{\prime} x_{0} y_{0}+2 \beta \phi^{\prime} x_{0} y_{0}^{\prime} .
\end{aligned}
$$

Note that the values of the invariants differ from the usual Courant-Snyder form by the presence of terms which are multiplied by $\phi^{\prime}$. Therefore, if at injection there is no magnetic field (i.e., $\phi^{\prime}=0$ ), the invariants coincide with the standard single particle emittances.

\section{BEAM TRANSPORT}

We now apply this theory to the beam transport; similar studies in presence of linear coupling are reported in $[12,13]$. We first consider a beam of nominal-momentum particles. Lossless beam transport requires that all along the transport line $r<R$, where $r=\sqrt{x^{2}+y^{2}}=$ $\sqrt{\beta\left[\epsilon_{1} \sin ^{2}\left(\psi+\delta_{1}\right)+\epsilon_{2} \sin ^{2}\left(\psi+\delta_{2}\right)\right]}$, and $R$ is the radius of the beam pipe. Since the sinusoidal terms are always $\leq 1$, one can estimate $r^{2}<\beta\left(\epsilon_{1}+\epsilon_{2}\right)$ and therefore one obtains a necessary (but not sufficient) condition for lossless transport which is much simpler:

$$
\epsilon_{1}+\epsilon_{2} \leq \frac{R^{2}}{\beta_{\max }},
$$

with $\beta_{\max }$ being the maximum $\beta$ over the channel length. Equation (20), together with Eq. (15), defines the volume occupied by the transported particles in the 4D laboratory phase space. All particles outside this volume might be lost. Another interesting application is the matching of two solenoidal channels: in order not to modify the two beta functions $\beta_{1}, \beta_{2}$ of the two channels, one needs to match the two optical functions. In this way, the beam will be transported from the end of the first channel to the beginning of the second channel without changing the single-particle invariants, and the condition for lossless transport (satisfied for each channel separately) will be maintained when they are joined together.

\section{A. Beam envelopes}

It is important to know the beam envelope. Unfortunately, it is not possible to predict analytically the envelope for any initial distribution. We first consider a distribution where all the particles have the same invariants $\epsilon_{1}, \epsilon_{2}$. We do not specify in which way particles should be distributed in $\delta_{1}, \delta_{2}$, but it is enough that the invariant surface is filled without holes. By direct integration we find that the volume of the invariant surface is $\pi^{2} \epsilon_{1} \epsilon_{2}$. We call this distribution matched invariants distribution. For this kind of distribution, by using Eqs. (17) we find the envelopes

$$
\begin{aligned}
X & =\sqrt{\beta \epsilon_{1}}|\mathfrak{c}|+\sqrt{\beta \epsilon_{2}}|\mathfrak{s}|, \\
Y & =\sqrt{\beta \epsilon_{2}}|\mathfrak{c}|+\sqrt{\beta \epsilon_{1}}|\mathfrak{g}|, \\
X^{\prime} & =\sqrt{\epsilon_{1} A / \beta}+\sqrt{\epsilon_{2} B / \beta}, \\
Y^{\prime} & =\sqrt{\epsilon_{2} A / \beta}+\sqrt{\epsilon_{1} B / \beta},
\end{aligned}
$$

where $A=\mathfrak{c}^{2}+\left(\alpha \mathfrak{c}+\phi^{\prime} \beta \mathfrak{g}\right)^{2}$ and $B=\mathfrak{g}^{2}+(\alpha \mathfrak{g}-$ $\left.\phi^{\prime} \beta \mathrm{c}\right)^{2}$. For this distribution the maximum radius of the beam is given by $r_{\max }=\sqrt{\beta\left(\epsilon_{1}+\epsilon_{2}\right)}$. The transverse kinetic energy for which $x^{\prime 2}+y^{\prime 2}$ is a measure is also an important quantity in designing an ionization cooling channel [14]. From Eqs. (17) we find that

$$
x^{\prime 2}+y^{\prime 2}=\left[\left(\alpha \mathrm{s}_{1}-\mathrm{c}_{1}\right)^{2}+\left(\phi^{\prime} \beta \mathrm{s}_{1}\right)^{2}\right] \epsilon_{1} / \beta+\left[\left(\alpha \mathrm{s}_{2}-\mathrm{c}_{2}\right)^{2}+\left(\phi^{\prime} \beta \mathrm{s}_{2}\right)^{2}\right] \epsilon_{2} / \beta+2 \phi^{\prime} \sqrt{\epsilon_{1} \epsilon_{2}} \sin \left(\delta_{2}-\delta_{1}\right) .
$$

Unfortunately, the expression for $E_{k} \equiv\left(x^{\prime 2}+y^{\prime 2}\right)_{\max }$ obtained from Eq. (22) is not simple. A practical formula is

$$
E_{k}=\frac{1}{2}\left(\gamma+\phi^{\prime 2} \beta+\sqrt{\gamma^{2}+\Omega}\right)\left(\epsilon_{1}+\epsilon_{2}\right)+\xi,
$$


where $\Omega=\left(\phi^{\prime 2} \beta\right)^{2}+2\left(\alpha^{2}-1\right) \phi^{\prime 2}$, and $\xi$ is a suitable number such that $0<\xi<2\left|\phi^{\prime}\right| \sqrt{\epsilon_{1} \epsilon_{2}}$. Note that this expression becomes the usual $\gamma\left(\epsilon_{1}+\epsilon_{2}\right)$ in absence of the solenoidal magnetic field. We compared Eqs. (21) with the numerical solution of the equations of motion in the CERN Neutrino Factory cooling channel [5]. One section is composed of four identical solenoids (radius $0.3 \mathrm{~m}$, length $0.76 \mathrm{~m}$, and $B_{0}=2 \mathrm{~T}$ ), spaced by $0.14 \mathrm{~m}$. The solenoid field $B_{s}(s)$ is represented by the field on axis; see [15]. The next section here is taken $0.48 \mathrm{~m}$ downstream. We show in Fig. 1 the trajectory of 30 particles injected at $0.62 \mathrm{~m}$ from the beginning of the first section, with invariants $\epsilon_{1}=$

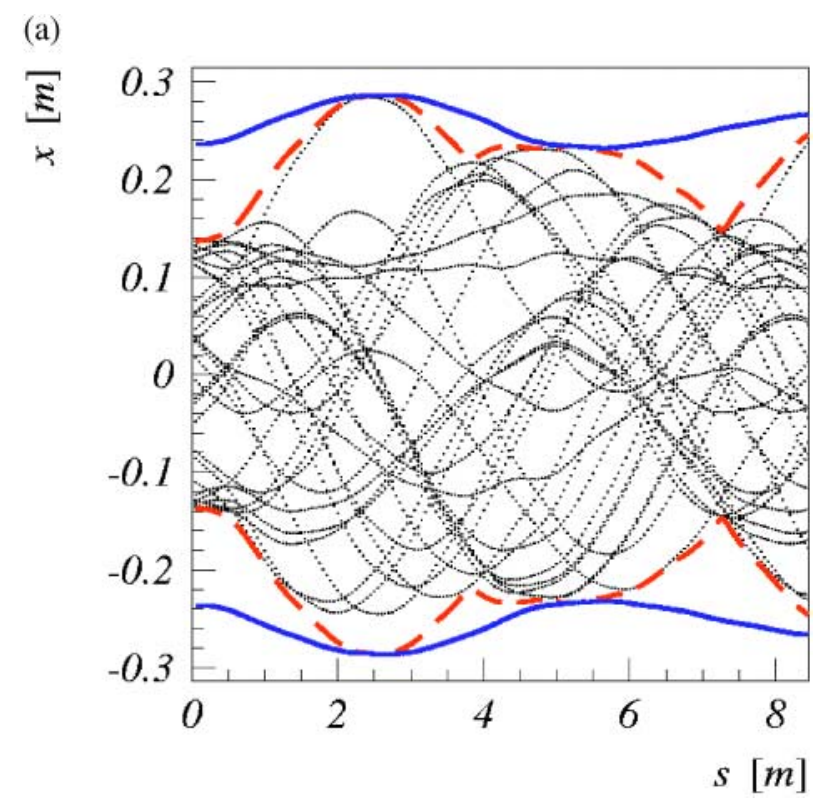

(b)

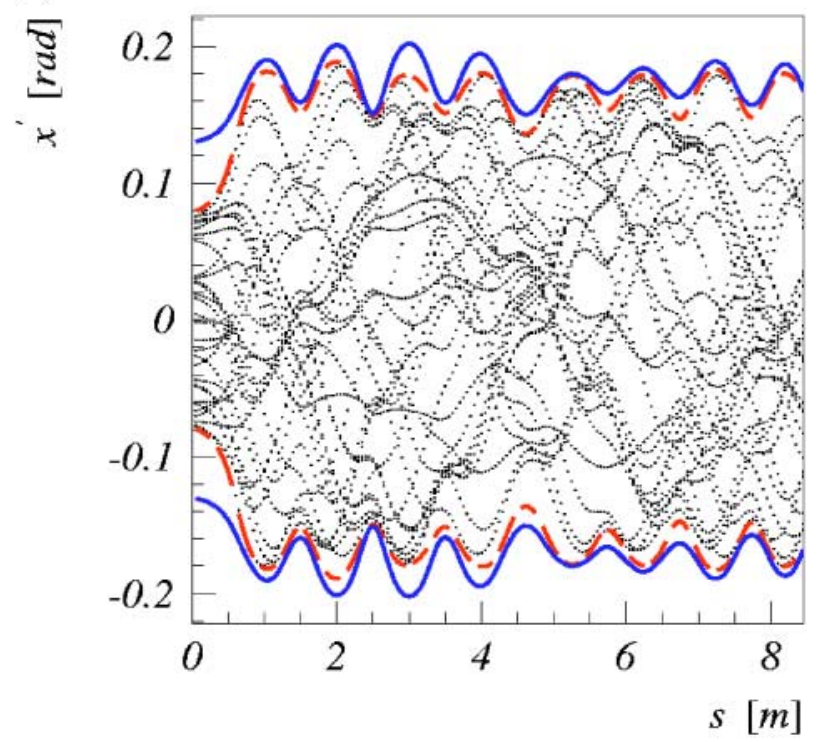

FIG. 1. (Color) (a) $r_{\max }$ (solid line), theoretical envelope $X$ (dashed line), and 30 particle trajectories (dotted lines). (b) $x^{\prime}$ vs $s$ (dotted lines), $X^{\prime}$ (dashed line), and $\sqrt{E_{k}}$ maximum (solid line) obtained for $\xi$ maximum.
$1 \mathrm{~cm} \mathrm{rad}, \epsilon_{2}=2 \mathrm{~cm} \mathrm{rad}$. The results are in complete agreement with the theoretical prediction: all the trajectories fit within the theoretical envelope. This method was used to find the matched condition for a lossless channel containing $44 \mathrm{MHz}$ cavities with a bore radius of $30 \mathrm{~cm}$.

Following [11] a more general matched invariants particle distribution can be written as $f\left(\tau^{2}\right)$ where $\tau^{2}=\epsilon_{1} / \epsilon_{10}+\epsilon_{2} / \epsilon_{20}$ and $f\left(\tau^{2}\right)$ is a function defined in $0<\tau^{2}<1$. This distribution satisfies the timeindependent Vlasov equation and it is therefore selfconsistent. The particle distribution is defined by $\epsilon_{10}, \epsilon_{20}$, and $f\left(\tau^{2}\right)$. Applying Eqs. (17) to this distribution we find the envelopes

$$
\begin{aligned}
X & =\sqrt{\beta\left(\epsilon_{10} \mathfrak{c}^{2}+\epsilon_{20} \mathfrak{S}^{2}\right)}, \\
Y & =\sqrt{\beta\left(\epsilon_{20} \mathfrak{c}^{2}+\epsilon_{10} \mathfrak{S}^{2}\right)}, \\
X^{\prime} & =\sqrt{\left(\epsilon_{10} A+\epsilon_{20} B\right) / \beta}, \\
Y^{\prime} & =\sqrt{\left(\epsilon_{20} A+\epsilon_{10} B\right) / \beta} .
\end{aligned}
$$

The maximum radius is $r_{\max }=\sqrt{\beta \max \left\{\epsilon_{10}, \epsilon_{20}\right\}}$, and we can express $E_{k} \equiv\left(x^{\prime 2}+y^{\prime 2}\right)_{\max }$ as

$$
E_{k}=\frac{1}{2}\left(\gamma+\phi^{\prime 2} \beta+\sqrt{\gamma^{2}+\Omega}\right) \max \left\{\epsilon_{10}, \epsilon_{20}\right\}+\xi,
$$

where $\xi$ is a suitable number such that $0<\xi<$ $\left|\phi^{\prime}\right| \sqrt{\epsilon_{10} \epsilon_{20}}$.

\section{B. Chromatic effects}

Calling $\delta=\left(p-p_{0}\right) / p_{0}$, for an off-momentum particle in a solenoidal channel, the Hamiltonian expanded up to the third order [8] leads to the equations of motion

$$
\begin{aligned}
& x^{\prime \prime}-S(1-\delta) y^{\prime}-\frac{S^{\prime}}{2}(1-\delta) y-\frac{S^{2}}{4} \delta^{2} x=0, \\
& y^{\prime \prime}+S(1-\delta) x^{\prime}+\frac{S^{\prime}}{2}(1-\delta) x-\frac{S^{2}}{4} \delta^{2} y=0 .
\end{aligned}
$$

As a first step toward the inclusion of the chromatic effects in the optics one can neglect the terms in $\delta^{2}$ so that the equations of motion are identical to Eqs. (1) but with a solenoidal strength $S=S_{0} /(1+\delta)$, where $S_{0}$ belongs to the reference particle. The equation of $\beta$ then becomes

$$
\frac{1}{2} \beta \beta^{\prime \prime}-\frac{1}{4} \beta^{\prime 2}+\frac{S_{0}^{2}}{4(1+\delta)^{2}} \beta^{2}=1 .
$$

Given one particle at the entrance of the solenoidal channel, from $\beta_{0}, \alpha_{0}$ in that position and $\delta$ we can find $\epsilon_{1}, \epsilon_{2}$ by using Eqs. (19). By using Eqs. (27) we can find $\beta_{\max }=$ $\beta_{\max }(\delta)$ over the channel length. Finally, from $\epsilon_{1}, \epsilon_{2}$, and $\beta_{\max }$ we find $r_{\max }$. If $r_{\max }>R$ the particle is lost. In this way an algorithm is obtained which in the $5 \mathrm{D}$ 
space $\left\{x, x^{\prime}, y, y^{\prime}, \delta\right\}$ defines the volume of initial conditions which is accepted by the solenoidal channel.

\section{Matching}

We can also apply this theory to match quadrupolar and solenoidal channels. Here the beginning and the end of the solenoidal channel satisfy the condition $\phi^{\prime}=0$. Consider a general matched invariants distribution in a quadrupolar transport channel [11]. This distribution is selfconsistent and, consequently, for each $\epsilon_{x 0}, \epsilon_{y 0}$ the plane $\left\{\delta_{1}, \delta_{2}\right\}$ is uniformly filled. The quadrupolar channel is merged to the solenoidal channel by means of a matching section which transforms the optic functions $\beta_{x}, \beta_{y}, \alpha_{x}, \alpha_{y}$ from the end of the quadrupolar channel to $\beta_{x, 1}, \beta_{y, 1}, \alpha_{x, 1}, \alpha_{y, 1}$ at the entrance of the solenoidal one. Matching is obtained when $\beta_{x, 1}=\beta_{y, 1}=\beta, \alpha_{x, 1}=$ $\alpha_{y, 1}=\alpha$. If these conditions are satisfied, the invariants of each particle are preserved, Eqs. (19) give $\epsilon_{1}=\epsilon_{x 0}, \epsilon_{2}=\epsilon_{y 0}$, and the initial distribution becomes a matched invariants distribution for the solenoidal channel [as confirmed by Eqs. (17)]. In the same way, one can match the solenoidal channel to a downstream quadrupolar channel. The transported particle distribution at the exit of the solenoidal channel is again the same self-consistent invariant matched distribution that was injected only when $\phi=\pi n$ with $n \in \mathbb{N}$. In fact, from Eqs. (17) for fixed $\epsilon_{1}, \epsilon_{2}$ a uniform distribution in $\left\{\delta_{1}, \delta_{2}\right\}$ (self-consistent) is invariant if $\phi=\pi n$ with $n \in \mathbb{N}$. This condition holds for each couple of $\epsilon_{1}, \epsilon_{2}$ of the matched invariants distribution at the end of the solenoidal channel and, consequently, the distribution injected into the downstream quadrupolar channel will be the same used at injection.

\section{D. rms invariants}

For ionization cooling it is important to measure the rms invariants and the effective volume in the $4 \mathrm{D}$ phase space from the particle distribution. However, in a solenoidal channel the two transverse planes are coupled and usual rms invariants are not preserved. The solenoidal channel rms invariants $\epsilon_{1,2 \mathrm{rms}}$ can be computed in the Larmor frame as

$$
\begin{aligned}
& \epsilon_{1 \mathrm{rms}}^{2}=\overline{w_{r}^{2}} \overline{w_{r}^{\prime 2}}-{\overline{w_{r} w_{r}^{\prime}}}^{2}, \\
& \epsilon_{2 \mathrm{rms}}^{2}=\overline{w_{i}^{2}} \overline{w_{i}^{\prime 2}}-{\overline{w_{i} w_{i}^{\prime}}}^{2} .
\end{aligned}
$$

By means of Eq. (3) these definitions can be easily implemented in a computer code where the beam tracking is performed in terms of the laboratory coordinates. For a matched invariants particle distribution $f\left(\tau^{2}\right)$ where $\tau^{2}=\epsilon_{1} / \epsilon_{10}+\epsilon_{2} / \epsilon_{20}$, the relation between $\epsilon_{10,20}$ and $\epsilon_{1,2 \mathrm{rms}}$ depends on $f$. Since the transformation Eq. (3) is volume preserving, the form of this distribution in the Larmor frame will not change and can be written again as $f\left(\tau^{2}\right)$ where now $\epsilon_{1}=\gamma w_{r}^{2}+2 \alpha w_{r} w_{r}^{\prime}+\beta w_{r}^{\prime 2}$, and $\epsilon_{2}=\gamma w_{i}^{2}+2 \alpha w_{i} w_{i}^{\prime}+\beta w_{i}^{\prime 2}$. This form has the same feature of the particle distributions used for rings [11] and allows a natural extension of usual particle distribution definitions at the solenoidal channel. In general, for a matched invariants particle distribution,

$$
\epsilon_{1,2 \mathrm{rms}}=C \epsilon_{10,20} \quad \text { where } C=\frac{1}{4} \frac{\int_{0}^{\infty} \tau^{5} f\left(\tau^{2}\right) d \tau}{\int_{0}^{\infty} \tau^{3} f\left(\tau^{2}\right) d \tau} .
$$

If $f\left(\tau^{2}\right) \propto \delta\left(\tau^{2}-1\right)$, we recover a KV-like distribution and $C=1 / 4$. If $f\left(\tau^{2}\right)=$ const in $0<\tau^{2}<1$ and $f\left(\tau^{2}\right)=0$ in $\tau^{2}>1$, we describe a waterbag-like distribution and $C=1 / 6$. For all the distributions such that $f\left(\tau^{2}\right)=0$ in $\tau^{2}>1$, the $4 \mathrm{D}$ volume which contains the distribution is $\left(\pi^{2} / 2\right) \epsilon_{10} \epsilon_{20}$.

\section{CONCLUSION}

We presented the solution of the single-particle linear optics in a solenoidal channel. We showed how to compute the single-particle invariants and found matched invariants distributions used to predict the beam envelopes. An algorithm to analyze the effect of off-momentum particles on lossless transport was proposed, and we gave a general rule to match solenoidal and quadrupolar channels. An rms invariants definition was presented, and we discussed the relation between rms invariants and the $4 \mathrm{D}$ phase space volume occupied by a matched invariants particle distribution.

\section{ACKNOWLEDGMENTS}

We wish to thank A. Lombardi, I. Hofmann, E. Todesco, and G. Turchetti for useful comments and discussion.

[1] D. V. Neuffer, Nucl. Instrum. Methods Phys. Res., Sect. A 350, 27 (1994).

[2] C. M. Ankenbrandt et al., Phys. Rev. ST Accel. Beams 2, 081001 (1999).

[3] H. Haseroth, in Proceedings of the Nufact Conference, Monterey CA (Elsevier Science, North-Holland, Amsterdam, 2000), p. 17.

[4] R. C. Fernow, in Proceedings of the 1999 Particle Accelerator Conference, New York (IEEE, Piscataway, NJ, 1999), p. 3020.

[5] A. Lombardi, CERN-NUFACT Note No. 34, 2000 (http: //molat.home.cern.ch/molat/neutrino/nfnotes.html).

[6] H. Wiedemann, Particle Accelerator Physics II (Springer, Berlin, 1995), Vol. 2, Chap. 3.

[7] D. A. Edwards and L.C. Teng, in Proceedings of the 1973 Particle Accelerator Conference, San Francisco, CA (IEEE, New York, 1973), p. 885.

[8] F. Willeke and G. Ripken, DESY Report No. 88/114, 1988.

[9] E. D. Courant and H.S. Snyder, Ann. Phys. (Paris) 3, 1 (1958).

[10] A. Bazzani, E. Todesco, G. Turchetti, and G. Servizi, CERN Report No. CERN-94-02, 1994. 
[11] M. Reiser, Theory and Design of Charged Particle Beams (Wiley, New York, 1994).

[12] R. K. Cooper, Part. Accel. 7, 41 (1975).

[13] D. Chernin, Part. Accel. 24, 29 (1988).
[14] K.-J. Kim and C.-X. Wang, Phys. Rev. Lett. 85, 760 (2000); G. Penn and J. S. Wurtele, ibid. 85, 764 (2000).

[15] J. A. Stratton, Electromagnetic Theory (McGraw-Hill, New York, 1941), p. 233. 